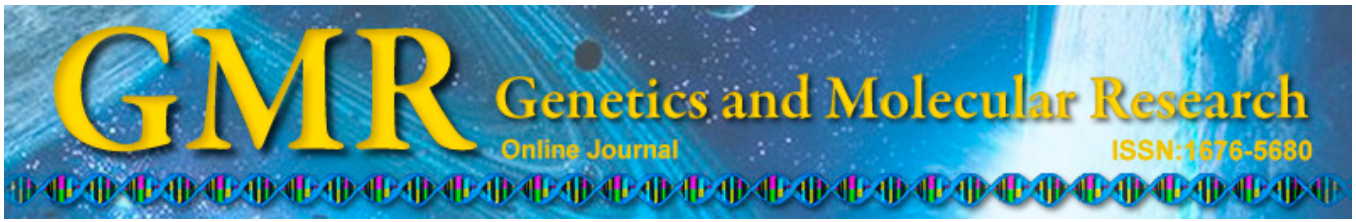

\title{
Impact of adherence to GOLD guidelines on 6-minute walk distance, MRC dyspnea scale score, lung function decline, quality of life, and quality-adjusted life years in a Shanghai suburb
}

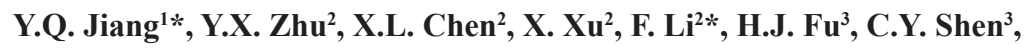 \\ Y.Y. Lu ${ }^{3}$, Q.J. Zhuang ${ }^{4}$, G. Xu' ${ }^{5}$, Y.Y. Cai ${ }^{6}$, Y. Zhang ${ }^{7}$, S.S. Liu ${ }^{8}$, \\ M.Y. Zhu ${ }^{8}$ and S.H. $\mathbf{L i}^{2}$ \\ 'Shanghai Municipal First People's Hospital Affiliated to Shanghai Jiaotong \\ University, Shanghai, China \\ ${ }^{2}$ Songjiang Branch of Shanghai Municipal First People's Hospital Affiliated to \\ Shanghai Jiaotong University, Shanghai, China \\ ${ }^{3}$ Xiaokunshan Town Primary Care of Songjiang District, Shanghai, China \\ ${ }^{4}$ Xinbang Town Primary Care of Songjiang District, Shanghai, China \\ ${ }^{5}$ School of Public Health, Shanghai Jiao Tong University, Shanghai, China \\ ${ }^{6}$ Institute of Respiratory Disease, Zhongshan Hospital Affiliated to Fudan \\ University, Shanghai, China \\ ${ }^{7}$ Shanghai Songjiang District Public Health Bureau, Shanghai, China \\ ${ }^{8}$ Shanghai Songjiang District Center for Diseases Control and Prevention, \\ Shanghai, China \\ *These authors contributed equally to this study. \\ Corresponding author: $\mathrm{F}$. Li \\ E-mail: fanlidoc@163.com \\ Genet. Mol. Res. 14 (3): 8861-8870 (2015) \\ Received December 4, 2014 \\ Accepted March 18, 2015 \\ Published August 3, 2015 \\ DOI http://dx.doi.org/10.4238/2015.August.3.9
}

ABSTRACT. We performed a 1-year cluster-randomized field trial to assess the effect of standardized management of chronic obstructive 
pulmonary disease (COPD) on lung function and quality of life (QOL) measures in patients in China. We used the Global Initiative for Chronic Obstructive Lung Disease (GOLD) treatment guidelines and assessed indexes including pulmonary function, QOL, quality-adjusted life years (QALY), Medical Research Council (MRC) dyspnea scale, 6-min walk distance (6-MWD), number of emergency visits, and frequency of hospitalization. Of a total of 711 patients with chronic cough and asthma, 132 were diagnosed as having COPD and 102 participated in this study [intervention group $(\mathrm{N}=47)$; control group $(\mathrm{N}=55)]$. We found that adherence to GOLD guidelines had a perceivable impact on 6-MWD, MRC dyspnea scale score, and QOL. The average QALY increased by 1.42 /person/year in the intervention group, but declined by $0.95 /$ person/year in the control group. We conclude that standardized management improves disease severity, QOL, and QALY in COPD patients when treatment protocols adhere to GOLD guidelines.

Key words: Chronic obstructive pulmonary disease; Cost effectiveness; General practitioner; Quality of life; Quality-adjusted life years

\section{INTRODUCTION}

Chronic obstructive pulmonary disease (COPD) is a common, preventable, and treatable disease characterized by persistent airflow obstruction [Global Initiative for Chronic Obstructive Lung Disease (GOLD), 2010]. World Health Organization statistics state that approximately 2.74 million people worldwide died of COPD in 2002; COPD is ranked as the fourth leading cause of death and is forecast to rank third by 2020. In addition, COPD ranks fifth in terms of economic burden. In China, the prevalence of COPD in people aged $>40$ years is $8.2 \%$ (Fang et al., 2011); the overall crude prevalence of COPD is $2.9 \%$ (Yin et al., 2011), and it is the third-most deadliest disease in the country (Yang et al., 2013).

Primary care is the mainstay of prevention and treatment of COPD (Ulrik et al., 2013). Patients in a stable phase of the disease are diagnosed and treated by primary care personnel. However, previous studies have reported that COPD patients do not receive standardized diagnoses and treatment in primary care (Yawn and Wollan, 2008; Jochmann et al., 2010; Li et al., 2013).

The GOLD was developed to increase awareness of and decrease morbidity and mortality from COPD (GOLD, 2010). The aims of GOLD are to improve the prevention and management of COPD through the concerted, worldwide efforts of people involved in all facets of health care and through provision of published guidance (GOLD, 2010).

For patients with asthma, some studies suggest that standardized guidelines can improve adherence to evidence-based treatment and reduce the financial burden of this disease (Jochmann et al., 2012). This situation may be similar for patients with COPD; however, there is an ongoing debate regarding the effectiveness of treatment protocols that conform to standardized guidelines.

Community health workers have paid considerable attention to the assessment of the outcomes of community intervention. This study sought to assess the changes in 6-min walk distance (6-MWD), Medical Research Council (MRC) dyspnea scale score, lung function decline, quality of life (QOL), and quality-adjusted life years (QALY) in COPD patients 
from two communities within the Songjiang District of Shanghai City, China, with different management modes.

\section{MATERIAL AND METHODS}

\section{Study design and subjects}

The study began in February 2008 and ended in April 2009. Using cluster randomization, two primary care groups within the Songjiang District of Shanghai City, China, were selected. Odd numbered centers were allocated to the intervention group (standardized management) and even numbered centers were allocated to the control group. The study inclusion criterion was a positive result in diagnostic pulmonary function tests, in accordance with GOLD guidelines (Rabe et al., 2007). Subjects with one or more of the following complications were excluded: severe heart, liver, kidney, or brain disease; poor language skills; inability to complete the study; and malignant tumor. After receiving information about the aim and main content of the research study, all patients gave their written informed consent. This study was approved by the Ethics Committee of the Songjiang Branch of Shanghai Municipal First People's Hospital, affiliated to Shanghai Jiaotong University. All patients included provided written documentation of informed consent.

\section{Data collection}

Patients were evaluated monthly by general practitioners. Assessment indexes included pulmonary function measurements [forced expiratory volume in $1 \mathrm{~s}$ (FEV1), percentage of predicted value of FEV1, and FEV1/forced vital capacity (FVC)], QOL, MRC dyspnea scale (GOLD, 2010) (five grades ranging from grade $0=$ mild to grade $5=$ severe), and 6-MWD. Other measures of pulmonary health included number of emergency visits, frequency of hospitalizations, and the cost associated with outpatient clinic and emergency room visits and hospitalization.

\section{Spirometry}

The height and weight of each patient was measured during the initial visit. MicroDL Spirometers (Microlab, UK) (Li et al., 2013) were used to measure FEV1, percentage of predicted value of FEV1, and FEV1/FVC at 20 min before and after inhalation of salbutamol $(400 \mu \mathrm{g})$. Patients were diagnosed as having COPD after exclusion of other diseases.

The severity of COPD was classified as follows (ATS Committee on Proficiency Standards for Clinical Pulmonary Function Laboratories, 2002): Stage I (mild), FEV1/FVC $<0.70$ and FEV $1 \geq 80 \%$ predicted; Stage II (moderate), FEV1/FVC $<0.70$ and FEV1 50-79\% predicted; Stage III (severe), FEV1/FVC $<0.70$ and FEV1 30-49\% predicted; and Stage IV (very severe), FEV1/FVC $<0.70$ and FEV $1<30 \%$ predicted.

\section{Quality of life}

The St. George's Respiratory Questionnaire (SGRQ) (Chinese version) (Jones et al., 1991, 1992), a standardized patient-completed questionnaire, was used to measure each patient's QOL. This questionnaire should be amended to accommodate differences in cultural traditions, languages, and living habits (Ferrer et al., 1996; Jones and Bosh, 1997; Lu et al., 
2003). However, a previous study showed that the SGRQ is a reliable and valid assessment of QOL in Chinese COPD patients; therefore, it was deemed appropriate for the present study (Lu et al., 2003). The SGRQ contains three sections: respiratory symptoms (cough, sputum production, and breathlessness), activity (walking uphill, getting dressed, sport, and housework), and impact on daily life (anxiety, pain, insecurity, and disappointment). SGRQ scores range from 0-100, with a score of zero indicating no impairment in QOL.

\section{Calculation of utility value and years of life lost based on the SGRQ}

Utility value was calculated using the following equation: (100 - total SGRQ score)/ 100. A utility value of 0.94 was determined previously in healthy Chinese subjects (He et al., 2009). A mean QOL score derived from a large population of healthy subjects calculated in a previous study (He et al., 2009) was used to calculate the utility value of Chinese COPD patients in the current study $(0.478 \pm 0.199$, calculated from total scores). Years of life lost were calculated by comparing the utility values of healthy subjects and COPD patients, excluding years of life lost because of early death. Years of life lost due to disability or injury were calculated to be 0.46 years of life per patient $(t=-23.317, \mathrm{P}=0.001)$.

Using life expectancy figures from the sixth national census in 2010 (National Bureau of Statistics of China, 2010), QALY was calculated as follows: life expectancy x utility value based on SGRQ scores.

\section{Management of COPD patients}

In accordance with GOLD, the COPD Prevention and Control Project for Community General Practitioners was formulated during the present study. It includes two aspects, namely, 1) training of general practitioners in COPD prevention and management, and COPD education of patients and their family members and 2) pharmaceutical guidelines for community health service centers.

\section{Knowledge training}

i) General practitioners received 4 training sessions that consisted of lectures and onsite demonstrations. Topics included strategies for smoking cessation, making a diagnosis of COPD and assessing the patient's condition, indications for and prescription of medications for COPD, rational use of home oxygen therapy, and pursed-lip and abdominal breathing. ii) Patients and their family members were educated by consultations, recordings, and lectures. They were also provided with information on curtailing smoking and recognition of COPD, and were trained in the use of inhalation equipment, methods of home oxygen therapy, and pursed-lip and abdominal breathing.

\section{Pharmaceutical guidelines}

Pharmaceutical guidelines for community health service centers in accordance with GOLD (2007 revision) (Jochmann et al., 2012) were as follows. i) Bronchodilators: Short-acting $\beta_{2}$-adrenergic receptor stimulants were prescribed 4 times daily, or as required. Anti-cholinergic aerosols were used 3 to 4 times daily, or as required. Based on the patient's condition, 
oral $\beta_{2}$-adrenergic receptor stimulants such as terbutaline and theophylline were provided. ii) Glucocorticoids: Grade III and IV patients received inhaled corticosteroids. Patients with recurrent acute exacerbations received $40 \mathrm{mg}$ oral prednisone daily for 5 consecutive days; if their symptoms were not controlled, they were sent to a higher-level hospital. iii) Antibiotic treatment: Antibiotics were used only for acute exacerbations of infection and occurrence of new infections. iv) Expectorants: Patients with viscous sputum or paranasal sinusitis were prescribed ambroxol $(30 \mathrm{mg}) 3$ times daily. Patients with little or no sputum did not receive expectorants. v) Antitussives: Provided to stable patients for regular use. vi) Other immunomodulators: Influenza vaccine was recommended. The patients gave their consent to all drug use.

\section{Community management of control group}

The general practitioners within the community received no training and their COPD patients were managed according to each general practitioner's practice. Patients with severe exacerbations of COPD were sent to a general hospital for treatment according to standards for transfer (Rabe et al., 2007).

\section{Follow-up}

Patients in both communities received monthly follow-up evaluations and the following data were recorded: cough, amount of sputum, color of sputum, shortness of breath, outpatient records, emergency visits and hospitalizations, drug use, and treatment-associated costs.

\section{Quality control}

In accordance with previously published standards (Li et al., 2013), a questionnaire was formulated. All investigators were trained, and specialized staff members performed quality control.

\section{Statistical analysis}

The data were analyzed using the SPSS 13.0 software (SPSS, Chicago, IL, USA). Enumerative data are reported as rates and measurement data are reported as means $\pm \mathrm{SD}$. The Student $t$-test was used to compare lung function, 6-MWD, MRC dyspnea scale scores, QOL scores, and QALY in the intervention and control groups. The rank sum test was used to compare cost-utility ratios between the two study groups. Multiple-linear regression analysis was employed to analyze the factors that influenced QALY. A value of $\mathrm{P}<0.05$ was considered to be statistically significant.

\section{RESULTS}

\section{General data}

According to the 2006 records of patients registered in the two study centers, lung function was assessed in 711 patients aged $\geq 40$ years with long-term chronic cough or asthma. Patients whose symptoms fitted with GOLD guideline diagnostic criteria (Rabe et al., 2007) 
were selected as subjects. Of the 711 patients with chronic cough and asthma, 132 (61 in the intervention group and 71 in the control group) were diagnosed as having COPD.

Within the 1-year follow-up period, 14/61 (23\%) COPD patients in the intervention group were lost to follow-up, including one with gastric cancer, one who died (accidental death), and one who developed pulmonary tuberculosis. The remaining 11 patients voluntarily withdrew from follow-up. Of the patients lost to follow-up, 5 had GOLD stage II, 4 had GOLD stage III, and 5 had GOLD stage IV COPD. Complete data were available for 47 subjects in the intervention group, including 22 men (47\%) and 25 women (53\%) aged 46-79 years (mean $\pm \mathrm{SD}, 69.1 \pm 7.8$ years). The duration of COPD ranged from $1-50$ years $(21.1 \pm 12.2$ years $)$.

In the control group, 16/71 (23\%) COPD patients were lost to follow-up. Four patients who were lost to follow-up died due to acute COPD exacerbations, 10 were lost to follow-up for unknown reasons, and 2 had incomplete data. The duration of COPD in the control group ranged from 1.5-56.5 years (19.8 \pm 12.4 years) (Tables 1 and 2$)$.

Table 1. Baseline data in the intervention and control groups.

\begin{tabular}{|c|c|c|c|c|}
\hline Variables & Intervention group & Control group & $t$ & $\mathrm{P}$ \\
\hline Age (years) & $69 \pm 6.44$ & $71 \pm 6.71$ & -1.843 & 0.068 \\
\hline Weight (kg) & $55.08 \pm 7.99$ & $54.87 \pm 10.52$ & 0.108 & 0.915 \\
\hline Height $(\mathrm{cm})$ & $160 \pm 6.05$ & $158 \pm 7.09$ & 1.189 & 0.237 \\
\hline FEV1 (L) & $0.96 \pm 0.49$ & $0.87 \pm 0.45$ & 1.007 & 0.316 \\
\hline 6-MWD (m) & $361.66 \pm 87.20$ & $398.07 \pm 107.59$ & -1.857 & 0.066 \\
\hline MRC dyspnea scale score & $0.4 \pm 0.71$ & $0.42 \pm 0.79$ & -0.93 & 0.926 \\
\hline SGRQ score & $46.96 \pm 22.17$ & $56.55 \pm 16.88$ & -2.425 & 0.017 \\
\hline
\end{tabular}

Data are reported as means \pm SD. Intervention group: Xiaokunshan Community Health Service Center; Control group: Xinbang Community Health Service Center; FEV1: forced expiratory volume in 1 s; 6-MWD: 6-min walk distance; MRC: Medical Research Council; SGRQ: St. George's Respiratory Questionnaire.

Table 2. Severity of COPD, gender, weight, and age of subjects at baseline and after 12 months.

\begin{tabular}{lcc}
\hline Variables & Baseline & 12 months \\
\hline Intervention group & & \\
Male [N (\%)] & $30(49.2 \%)$ & $22(46.8 \%)$ \\
Female [N (\%)] & $31(50.8 \%)$ & $25(53.2 \%)$ \\
Age (years \pm SD) & $69 \pm 6.44$ & $69 \pm 8.00$ \\
Height (cm \pm SD) & $160 \pm 6.05$ & $160 \pm 5.90$ \\
Current smokers [N (\%)] & $5(8.2 \%)$ & $2(4.3 \%)$ \\
COPD GOLD I [N (\%)] & $4(6.6 \%)$ & $3(6.4 \%)$ \\
COPD GOLD II [N (\%)] & $14(23.0 \%)$ & $8(17.0 \%)$ \\
COPD GOLD III [N (\%)] & $28(46.0 \%)$ & $25(53.2 \%)$ \\
COPD GOLD IV [N (\%)] & $15(24.6 \%)$ & $11(23.4 \%)$ \\
Control group & & $37(67.3 \%)$ \\
Male [N (\%)] & $45(63.4 \%)$ & $18(32.7 \%)$ \\
Female [N (\%)] & $26(36.6 \%)$ & $72 \pm 9.14$ \\
Age (years \pm SD) & $71 \pm 6.71$ & $159 \pm 7.17$ \\
Height (cm \pm SD) & $158 \pm 7.09$ & $19(34.5 \%)$ \\
Current smokers [N (\%)] & $19(31.1 \%)$ & $1(1.9 \%)$ \\
COPD GOLD I [N (\%)] & $2(2.8 \%)$ & $18(32.7 \%)$ \\
COPD GOLD II [N (\%)] & $15(21.1 \%)$ & $21(38.2 \%)$ \\
COPD GOLD III [N (\%)] & $30(42.3 \%)$ & $15(27.3 \%)$ \\
COPD GOLD IV [N (\%)] & $24(33.8 \%)$ & . \\
\hline
\end{tabular}

Intervention group: Xiaokunshan Community Health Service Center; Control group: Xinbang Community Health Service Center; COPD: chronic obstructive pulmonary disease; GOLD: Global Initiative for Chronic Obstructive Lung Disease. 
A significant difference between the intervention group and the control group was observed in 6-MWD and QOL scores, which were higher in the control group vs the intervention group at baseline $(\mathrm{P}<0.05$; Table 3$)$. Other assessments showed no differences between the intervention group and the control group.

Differences in pulmonary function, QOL, 6-MWD, and MRC dyspnea scale scores in the intervention and control groups from baseline to 12 months are summarized in Table 4 .

\begin{tabular}{|c|c|c|c|c|c|c|c|c|}
\hline \multirow[t]{2}{*}{ Variables } & \multicolumn{4}{|c|}{ Intervention group } & \multicolumn{4}{|c|}{ Control group } \\
\hline & Baseline & 12 months & $t$ & $P$ & Baseline & 12 months & $t$ & $P$ \\
\hline FEV1 (L) & $0.96 \pm 0.49$ & $0.88 \pm 0.43$ & 1.623 & 0.111 & $0.87 \pm 0.44$ & $0.87 \pm 0.42$ & -1.53 & 0.131 \\
\hline QOL score & $47 \pm 22$ & $39 \pm 20$ & 3.215 & 0.002 & $57 \pm 17$ & $62 \pm 18$ & -2.879 & 0.006 \\
\hline 6-MWD (m) & $362 \pm 87$ & $354 \pm 89$ & 0.76 & 0.451 & $398 \pm 108$ & $351 \pm 94$ & 4.913 & 0.000 \\
\hline MRC dyspnea scale score & $0.40 \pm 0.71$ & $0.34 \pm 0.64$ & 0.535 & 0.595 & $0.55 \pm 0.79$ & $0.84 \pm 0.98$ & -1.765 & 0.083 \\
\hline
\end{tabular}

Data are reported as means \pm SD. Intervention group: Xiaokunshan Community Health Service Center; Control group: Xinbang Community Health Service Center; FEV1: forced expiratory volume in $1 \mathrm{~s}$; QOL: quality of life; 6-MWD: 6-min walk distance; MRC: Medical Research Council.

Table 4. Differences in pulmonary function, quality of life, 6-min walk distance, and MRC dyspnea scale scores in the intervention and control groups between baseline and 12 months.

\begin{tabular}{lcccc}
\hline Group & FEV1 (L) & QOL score & 6-MWD $(\mathrm{m})$ & MRC dyspnea scale score \\
\hline Intervention & $-0.08 \pm 0.35$ & $-8 \pm 17$ & $-7 \pm 67$ & $-0.06 \pm 0.82$ \\
Control & $0.00 \pm 0.16$ & $6 \pm 14$ & $-45 \pm 70$ & $0.29 \pm 0.88$ \\
$t$ & -1.55 & -4.36 & 2.73 & -2.10 \\
$\mathrm{P}$ & 0.146 & 0.000 & 0.008 & 0.038 \\
\hline
\end{tabular}

Data are reported as means \pm SD. Intervention group: Xiaokunshan Community Health Service Center; Control group: Xinbang Community Health Service Center; FEV1: forced expiratory volume in $1 \mathrm{~s}$; QOL: quality of life; 6-MWD: 6-min walk distance; MRC: Medical Research Council.

\section{Assessment of quality of life and QALY}

Significant differences in SGRQ scores were observed between the two study groups, both before and after intervention $(\mathrm{P}<0.05)$. The SGRQ score decreased in the intervention group but increased in the control group. The mean QALY increased by 1.42/person/year in the intervention group, but declined by $0.95 /$ person/year in the control group (Table 5).

Table 5. Comparison of quality of life and quality-adjusted life years between the groups.

\begin{tabular}{|c|c|c|c|c|c|c|c|}
\hline \multirow[t]{2}{*}{ Group } & \multicolumn{2}{|c|}{ SGRQ } & \multicolumn{2}{|c|}{ Weight coefficient } & \multicolumn{2}{|c|}{ QALY } & \multirow{2}{*}{$\begin{array}{c}\text { Gained QALY } \\
12 \text { months - baseline }\end{array}$} \\
\hline & Baseline & 12 months & Baseline & 12 months & Baseline & 12 months & \\
\hline Intervention & $46.96 \pm 22.17$ & $39.13 \pm 19.78$ & $0.53 \pm 0.22$ & $0.61 \pm 0.20$ & $10.18 \pm 7.09$ & $11.59 \pm 7.24$ & $1.42 \pm 3.11$ \\
\hline Control & $56.55 \pm 16.88$ & $62.11 \pm 14.88$ & $0.43 \pm 0.17$ & $0.38 \pm 0.15$ & $7.17 \pm 5.76$ & $6.22 \pm 5.11$ & $0.95 \pm 2.11$ \\
\hline$t$ & -2.425 & -6.539 & 2.425 & 6.539 & 2.364 & 4.262 & 4.411 \\
\hline$P$ & 0.017 & 0 & 0.017 & 0 & 0.02 & 0 & 0 \\
\hline
\end{tabular}

Data are reported as means \pm SD. Intervention group: Xiaokunshan Community Health Service Center; Control group: Xinbang Community Health Service Center; SGRQ: St. George's Respiratory Questionnaire; QALY: quality-adjusted life years. 
Multiple linear regression analysis with QALY as the dependent variable showed that scores for the SGRQ correlated positively with QALY and this correlation was statistically significant (Table 6).

Table 6. Factors influencing quality-adjusted life years (multiple linear regression with quality-adjusted life years as the dependent variable).

\begin{tabular}{|c|c|c|c|c|c|c|}
\hline Item & B partial regression & Standardized partial & $t$ & $P$ & \multicolumn{2}{|c|}{$95 \% \mathrm{CI}$ for $\mathrm{B}$} \\
\hline Constant & -0.506 & & -0.463 & 0.644 & -2.675 & 1.662 \\
\hline Pre-intervention SGRQ activity score & 0.055 & 0.465 & 5.355 & 0 & 0.035 & 0.076 \\
\hline Post-intervention total score of quality of life & -1.368 & -0.24 & -2.757 & 0.007 & -2.352 & -0.383 \\
\hline
\end{tabular}

SGRQ: St. George's Respiratory Questionnaire.

\section{DISCUSSION}

We sought to assess the impact of adherence to GOLD guidelines by general practitioners when treating COPD patients over a 12-month follow-up period. Guidelines serve to standardize disease management, with the aim of improving patient care. The benefits of treatment for COPD according to GOLD guidelines are a reduction in the number of exacerbations, a delay in the loss of lung function, and an improvement in QOL (GOLD, 2010; Fang et al., 2011). Treatment algorithms are tailored to GOLD stages with the aim of improving outcomes in patients according to the severity of their disease.

The present study compared 6-MWD, MRC dyspnea scale score, lung function decline, QOL, and QALY after a 12-month follow-up period in a COPD patient group treated with adherence to the GOLD guidelines (intervention group) $v s$ a group treated according to the general practitioner's previous practice (control group).

Although the present study found no significant improvement in COPD patients' pulmonary function following GOLD guideline-based management, a significant improvement in 6-MWD was observed between baseline and month 12 of follow-up. In addition, significant differences were seen between the intervention and control groups in improvement in 6-MWD and MRC dyspnea scale score between baseline and month 12 of follow-up, suggesting that the intervention group fared significantly better than the control group.

In line with changes in the medical model, increasing numbers of clinical studies are addressing QOL (Calverley et al., 2007; Gunasekera et al., 2008; He et al., 2009). The SGRQ assessment tool, a disease-specific questionnaire that provides a measure for QOL, is now widely used (Calverley et al., 2007) in COPD patients both outside China (Ferrer et al., 1996; Jones and Bosh, 1997) and within China (Lu et al., 2003). Significant differences between the intervention and control groups were observed for improvement in QOL in the present study.

QALY, a measure of disease burden that encompasses QOL and life expectancy, is used to assess the economic value of medical intervention (Jones and Bosh, 1997). QOL is indicated by a number between 0 and 1 (death $=0$; full health $=1)$. The QALY score combines this QOL index with years of survival; its number represents the number of years in perfect health. Thus, 1 year of perfect health scores 1 in the QALY assessment. The current study showed that management of COPD according to GOLD guidelines by general practitioners in primary care improves the cost effectiveness of treatment. The average QALY increased by $1.42 /$ person/year in the intervention group but declined by $0.95 /$ person/year in 
the control group.

Of the demographic factors analyzed (gender, age, QOL, pulmonary function, MRC dyspnea scale score, and 6-MWD), two factors influenced QALY: scores reflecting the severity and control of the patients' conditions pre-intervention and the choice of medical interventions by general practitioners. Thus, the specific medical interventions performed by general practitioners are key factors determining the QALY scores of COPD patients.

Currently, patients with COPD do not make optimal use of community health services (Lu et al., 2003; Oba, 2007). Increasing COPD patient participation in community medical care and distributing patient information to help patients understand and more effectively use community health services should therefore be a major goal for the Health Authority.

\section{Limitations of this study}

1) A high rate of loss to follow-up; 2) a small sample size; and 3) baseline differences in QOL scores between the two groups. At the 1-year follow-up, there was a significant improvement in QOL scores in the intervention group and a significant difference in QOL scores between the control and intervention groups. Thus, the results of this study are clinically significant.

\section{CONCLUSIONS}

Adherence to the GOLD guidelines in primary care is effective and feasible, and improves 6-MWD, MRC dyspnea scale score, and QOL in COPD patients. In addition, medical interventions according to the GOLD guidelines decrease socioeconomic burden, achieve optimal cost utility, and encourage rational use of medical health resources for COPD patients.

\section{Conflicts of interest}

The authors declare no conflict of interest.

\section{ACKNOWLEDGMENTS}

We are very grateful to Boehringer Ingelheim Pharmaceuticals in China for providing the SGRQ (Chinese version) and statistical analysis software. Research supported by the Shanghai Municipal Health Bureau Project (\#2007169) and the Shanghai Municipal Science Committee (\#124119b0700).

\section{REFERENCES}

ATS Committee on Proficiency Standards for Clinical Pulmonary Function Laboratories (2002). ATS statement: Guidelines for the six-minute walk test. Am. J. Respir. Crit. Care Med. 166: 111-117.

Calverley PM, Anderson JA, Celli B, Ferguson GT, et al. (2007). TORCH investigators. Salmeterol and fluticasone propionate and survival in chronic obstructive pulmonary disease. N. Engl. J. Med. 356: 775-789.

Fang XC, Wang C and Bai CX (2011). COPD in China: The burden and importance of proper management. Chest 139: 920-929.

Ferrer M, Alonso J, Prieto L, Plaza V, et al. (1996). Validity and reliability of the St. George's Respiratory Questionnaire after adaptation to a different language and culture: the Spanish example. Eur. Respir. J. 9: 1160-1166.

Global Initiative for Chronic Obstructive Lung Disease (GOLD) (2010). Global Strategy for the Diagnosis, Management, 
and Prevention of COPD, 2010. Available at [http://www.goldcopd.org/uploads/users/files/GOLDReport April112011.pdf]. Accessed July 28, 2011.

Gunasekera S, Fraser J and Alexander C (2008). Quality of life in Hepatitis C virus infection: assessment of rural patients living in north-western New South Wales. Aust. J. Rural Health 16: 213-220.

He QY, Zhou X, Xie CM, Liang ZA, et al. (2009). Impact of chronic obstructive pulmonary disease on quality of life and economic burden in Chinese urban areas. Zhonghua Jie He He Hu Xi Za Zhi 32: 253-257.

Jochmann A, Neubauer F, Miedinger D, Schafroth S, et al. (2010). General practitioner's adherence to the COPD GOLD guidelines: baseline data of the Swiss COPD Cohort Study. Swiss Med. Wkly. Doi: 104414/smw.2010.13053.

Jochmann A, Scherr A, Jochmann DC, Miedinger D, et al. (2012). Impact of adherence to the GOLD guidelines on symptom prevalence, lung function decline and exacerbation rate in the Swiss COPD cohort. Swiss Med. Wkly. 142: w13567.

Jones PW and Bosh TK (1997). Quality of life changes in COPD patients treated with salmeterol. Am. J. Respir. Crit. Care Med. 155: 1283-1289.

Jones PW, Quirk FH and Baveystock CM (1991). The St. George's Respiratory Questionnaire. Respir. Med. 85 (Suppl B): 25-31; discussion 33-37

Jones PW, Quirk FH, Baveystock CM and Littlejohns P (1992). A self-complete measure of health status for chronic airflow limitation. The St. George's Respiratory Questionnaire. Am. Rev. Respir. Dis. 145: 1321-1327.

Li F, Cai Y, Zhu Y, Chen X, et al. (2013). The evaluation of general practitioners' awareness/knowledge and adherence to the GOLD guidelines in a Shanghai suburb. Asia Pac. J. Public. Health. Doi: 10.1177/1010539513475654.

Lu WX, Zhang YJ, Hu B, Ma Y, et al. (2003). Application of St George's respiratory questionnaire in evaluating the life quality of Chinese patients with chronic obstructive pulmonary disease. Zhonghua Jie He He Hu Xi Za Zhi 26: 195198.

National Bureau of Statistics of China (2010). The 6th national census data bulletin (No. 1). http://www.stats.gov.cn/tjfx/ jdfx/t20110428_402722253.htm.

Oba Y (2007). Cost-effectiveness of long-acting bronchodilators for chronic obstructive pulmonary disease. Mayo Clin. Proc. 82: 575-582.

Rabe KF, Hurd S, Anzueto A, Barnes PJ, et al. (2007). Global Initiative for Chronic Obstructive Lung Disease. Global strategy for the diagnosis, management, and prevention of chronic obstructive pulmonary disease: GOLD executive summary. Am. J. Respir. Crit. Care Med. 176: 532-555.

Ulrik CS, Sørensen TB, Højmark TB, Olsen KR, et al. (2013). Adherence to COPD guidelines in general practice: impact of an educational programme delivered on location in Danish general practices. Prim. Care Respir. J. 22: 23-28.

Yang G, Wang Y, Zeng Y, Gao GF, et al. (2013). Rapid health transition in China, 1990-2010: findings from the Global Burden of Disease Study 2010. Lancet 381: 1987-2015.

Yawn BP and Wollan PC (2008). Knowledge and attitudes of family physicians coming to COPD continuing medical education. Int. J. Chron. Obstruct. Pulmon. Dis. 3: 311-317.

Yin P, Zhang M, Li Y, Jiang Y, et al. (2011). Prevalence of COPD and its association with socioeconomic status in China: findings from China Chronic Disease Risk Factor Surveillance 2007. BMC Public Health 11: 586. 